\title{
Plasmodium falciparum msp1, msp2 and glurp allele frequency and diversity in sub-Saharan Africa
}

\author{
Felista Mwingira ${ }^{1,2}$, Gamba Nkwengulila ${ }^{2}$, Sonja Schoepflin ${ }^{3,4}$, Deborah Sumari ${ }^{5}$, Hans-Peter Beck ${ }^{3,4}$, \\ Georges Snounou ${ }^{6}$, Ingrid Felger ${ }^{3,4}$, Piero Olliaro $^{7,8}$ and Kefas Mugittu ${ }^{5^{*}}$
}

\begin{abstract}
Background: The efficacy of anti-malarial drugs is assessed over a period of 28-63 days (depending on the drugs' residence time) following initiation of treatment in order to capture late failures. However, prolonged follow-up increases the likelihood of new infections depending on transmission intensity. Therefore, molecular genotyping of highly polymorphic regions of Plasmodium falciparum msp1, msp2 and glurp loci is usually carried out to distinguish recrudescence (true failures) from new infections. This tool has now been adopted as an integral part of anti-malarial efficacy studies and clinical trials. However, there are concerns over its utility and reliability because conclusions drawn from molecular typing depend on the genetic profile of the respective parasite populations, but this profile is not systematically documented in most endemic areas. This study presents the genetic diversity of P. falciparum msp1, msp2 and glurp markers in selected sub-Saharan Africa countries with varying levels of endemicity namely Malawi, Tanzania, Uganda, Burkina Faso and São Tomé.

Methods: A total 780 baseline (Day 0) blood samples from children less than seven years, recruited in a randomized controlled clinical trials done between 1996 and 2000 were genotyped. DNA was extracted; allelic frequency and diversity were investigated by PCR followed by capillary electrophoresis for msp2 and fragment sizing by a digitalized gel imager for msp1 and glurp.

Results and Conclusion: Plasmodium falciparum msp1, msp2 and glurp markers were highly polymorphic with low allele frequencies. A total of 17 msp1 genotypes [eight MAD20-, one RO33- and eight K1-types]; 116 msp2 genotypes [83 3D7 and 33 FC27-types] and 14 glurp genotypes were recorded. All five sites recorded very high expected heterozygosity $\left(H_{E}\right)$ values $(0.68-0.99)$. $H_{E}$ was highest in msp2 locus $\left(H_{E}=0.99\right)$, and lowest for msp1 $\left(H_{E}\right.$ $=0.68)(P<0.0001)$. The genetic diversity and allelic frequency recorded were independent of transmission intensity $(P=0.84, P=0.25$ respectively. A few genotypes had particularly high frequencies; however the most abundant showed only a $4 \%$ probability that a new infection would share the same genotype as the baseline infection. This is unlikely to confound the distinction of recrudescence from new infection, particularly if more than one marker is used for genotyping. Hence, this study supports the use of msp 1, msp2 and glurp in malaria clinical trials in sub-Saharan Africa to discriminate new from recrudescent infections.
\end{abstract}

\section{Background}

The efficacy of anti-malarial drugs in endemic areas is assessed over a follow-up period of 28 to 63 days, depending of the drug's residence time in the organism, following initiation of treatment. While a longer followup allows capturing more late failures [1] the likelihood

\footnotetext{
* Correspondence: kmugittu@ihi.or.tz

${ }^{5}$ Ifakara Health Institute, P. O. Box 74 Bagamoyo, Tanzania

Full list of author information is available at the end of the article
}

of re-infection increases in a way that is dependent upon the intensity of transmission in the study area. Comparing molecular genotypic pattern of pre-treatment (baseline) and recurrent infections provides a means to help characterize the recurrent parasites as a recrudescence, i.e. a true failure, or a new infection (either from pre-existing liver infection or a newly established infection from an infected mosquito bite), i.e. a successful treatment.

\section{Ciomed Central}

(ㄷ) 2011 Mwingira et al; licensee BioMed Central Ltd. This is an Open Access article distributed under the terms of the Creative Commons Attribution License (http://creativecommons.org/licenses/by/2.0), which permits unrestricted use, distribution, and reproduction in any medium, provided the original work is properly cited. 
Several Plasmodium falciparum genes show extensive genetic polymorphism. This phenomenon is exploited for genetic finger printing and for assessing parasite population dynamics. For instance, high polymorphism has been shown in msp1, msp 2 and glurp genes in different geographical locations in malaria endemic areas [2-7]. The probability of a patient, particularly in areas of intense transmission, to be newly infected with a parasite possessing an identical genotype to the former infection is low [8]. Therefore, comparing the genotypes of the three loci at baseline and at the time of parasite recurrence would be expected to discriminate between recrudescent and new infections $[8,9]$. Numerous clinical drug trials have applied this approach to correct the outcomes of drug efficacy studies [10-17].

However, the discriminating power of different markers is dependent on the extent of allelic diversity and on the frequency of each allele within the population under study. Indeed, recurrent episodes after treatment can be reliably classified as recrudescence or re-infections if the frequencies of the $m s p 2, m s p 1$, and glurp alleles, as detected by the genotyping protocol employed, are known. It is obvious that a second infection appearing during follow-up after the first has been apparently cleared can be erroneously classified as recrudescent if some alleles predominate in the population or if heterozygosity is low, because under these circumstances a new infection would be more likely to share the same genotype as the baseline infection. This will lead to an over-estimation of treatment failures and consequently unnecessary treatment policy changes.

The allelic diversity of $m s p 2$ has been observed to be high in some areas, such as in the Kilombero valley in Tanzania (82 msp2 alleles) [2], Papua New Guinea (42 msp2 alleles) [18], Ghana (164 msp2 alleles) [19] and Côte d'Ivoire (50 msp2 genotypes) [20]. Conversely, there is very limited information on $m s p 1$ or glurp diversity across sub-Saharan Africa. This study presents the $m s p 1, m s p 2$ and glurp genetic diversity and allele frequencies in five Sub-Saharan African countries with different transmission intensities namely, Malawi, Tanzania, Uganda, Burkina Faso and São Tomé.

\section{Methods}

\section{Study area and design}

This study is part of two artemisinin combination therapy (ACT) trials that took place in sub-Saharan African regions with different malaria transmission intensities as detailed below;

(i) Randomized, double-blinded artemisinin-combination trials sponsored by the UNICEF/UNDP/ WORLD BANK/WHO Special Programme for Research and Training in Tropical Diseases (WHO/
TDR) between 1999 and 2000 in Burkina Faso, Gabon, The Gambia, São Tomé, Senegal, Uganda, Malawi and Kenya. The drugs tested in these trials were amodiaquine, sulphadoxine-pyrimethamine (SP) or chloroquine (CQ) alone or in combination with artesunate. Due to time and financial limitations only a subset of samples were analysed from only four of these countries (Malawi, Uganda, Burkina Faso and São Tomé) and used in the current study.

(ii) A trial conducted by the Ifakara Health Institute (IHI) and Swiss Tropical and Public Health Institute (Swiss TPH) in Tanzania in 1996. Drugs tested in this study were CQ alone vs. artemether-lumefantrine (AL).

The study site characteristics, methodological and clinical findings are described elsewhere [13,21,22]. For all the studies ethical approval was obtained from relevant local and external ethics committees. Prior to recruitment, informed consent was obtained from the children's parents or guardians. Blood samples from participant patients were collected on IsoCode ${ }^{\mathrm{TM}}$ Stix (in WHO/TDR trials) or as whole blood (in IHI/Swiss TPH trial) on day $0,7,14,21$ and 28. A total of 780 baseline pre-treatment samples (Day 0) were randomly selected for genotyping, $\mathrm{n}=180$ from each study site except Tanzania $(\mathrm{n}=60)$.

\section{Molecular genotyping}

DNA was extracted from IsoCode ${ }^{\mathrm{TM}}$ Stix (at IHI) by boiling (for the WHO/TDR trial samples) as described by manufacturer or by the standard phenol-chloform method (at Swiss TPH) from whole blood (for IHI/ Swiss TPH trial samples) and finally suspended in $50 \mu \mathrm{l}$ or $30 \mu \mathrm{l}$ of double-distilled sterile water, respectively. The template was kept frozen until needed. PCR amplification of template DNA and analysis of region II of glurp, central polymorphic region of $m s p 2$ (3D7 and FC27 allelic families), and block 2 of $m s p 1$ (K1, MAD20 and RO33 allelic families) was performed in accordance to the recently recommended genotyping protocol [23] with minor modifications, i.e. the primary PCR amplification for glurp and $m s p 2$ was duplexed whereas $m s p 1$ and all nested amplifications were uniplexed.

The primary duplexed and uniplexed PCR amplifications of were carried out in $75 \mu \mathrm{l}$ whereas all nested reactions were uniplexed and done in $50 \mu$ final reaction volumes. The primary duplexed (glurp and $m s p 2$ ) reactions contained $1 \mathrm{x}$ PCR buffer B (Firepol Solis ${ }^{\circledR}$ ), $1.57 \mathrm{mM} \mathrm{MgCl}_{2}, 120 \mu \mathrm{M}$ of each dNTP, 0.4 units of Taq DNA polymerase (Firepol Solis ${ }^{\circledR}$ ), $30 \mu \mathrm{M}$ of each oligonucleotide primer (Operon, Table 1) and $5 \mu \mathrm{l}$ of template DNA. 
Table 1 Name, sequence and amplicon sizes generated by Plasmodium falciparum msp1, msp2 and glurp PCR amplification primers

\begin{tabular}{|c|c|c|c|}
\hline Gene & Amplification & Primer & Primer sequence \\
\hline \multirow[t]{8}{*}{ mspl } & Primary & M1OR & 5'cttaaatagtattctaattcaagtggatca 3' \\
\hline & & MIOF & 5'ctagaagctttagaagatgcagtattg 3' \\
\hline & Secondary & MKR & 5'gcttgcatcagctggagggcttgcaccaga 3' \\
\hline & & MKF & 5'aaatgaagaagaaattactacaaaaggtgc 3' \\
\hline & Secondary & M1MR & 5'atctgaaggatttgtacgtcttgaattacc 3' \\
\hline & & M1MF & 5'aaatgaaggaacaagtggaacagctgttac 3' \\
\hline & Secondary & M1RF & 5'taaaggatggagcaaatactcaagttgttg 3' \\
\hline & & $\mathrm{RO} 33 \mathrm{R} 2$ & $\begin{array}{l}5^{\prime} \text { caagtaattttgaactctatgttttaaatcagcgta } \\
3^{\prime}\end{array}$ \\
\hline \multirow[t]{5}{*}{ msp2 } & Primary & $\mathrm{S} 2$ & 5'-gaaggtaattaaaacattgtc $3^{\prime}$ \\
\hline & & S3 & $5^{\prime}$-gagggatgttgctgctccacag $3^{\prime}$ \\
\hline & Secondary & $\begin{array}{l}\text { S1TAIL } \\
\text { FW }\end{array}$ & 5'-gcttataatatgagtataaggagaa 3' \\
\hline & & $\begin{array}{l}\text { M5- } \\
\text { FC27-RV }\end{array}$ & 6FAM 5'-gcattgccagaacttgaa 3' \\
\hline & & $\begin{array}{l}\text { N5-3D7- } \\
\text { RV }\end{array}$ & VIC 5'-ctgaagaggtactggtaga 3' \\
\hline \multirow[t]{3}{*}{ glurp } & Primary & GF3 & 5'acatgcaagtgtgatcctgaa 3' \\
\hline & & $\mathrm{GF}^{*}$ & 5'tgtaggtaccacgggttcttgtgg $3^{\prime}$ \\
\hline & Secondary & GNF & $5^{\prime}$ tgttcacactgaacaattagatttagatca $3^{\prime}$ \\
\hline
\end{tabular}

Note: ${ }^{*}$ used also for as reverse primer in the secondary amplification.

The nested glurp amplification final reaction volumes contained $1 \mathrm{x}$ buffer B, $1.5 \mathrm{mM} \mathrm{MgCl}_{2}, 128 \mu \mathrm{M}$ of each dNTP, $75 \mu \mathrm{M}$ of each oligonucleotide primer, 0.4 units of Taq DNA polymerase (Firepol Solis ${ }^{\circledR}$ ) and $2 \mu \mathrm{l}$ of primary PCR product as a template whereas nested msp 2 reactions had $1 \mathrm{x}$ buffer $\mathrm{B}, 1.5 \mathrm{mM} \mathrm{MgCl}_{2}, 200 \mu \mathrm{M}$ of each dNTP, $300 \mu \mathrm{M}$ of each fluorescent labeled familyspecific oligonucleotide primers (Applied Biosystems) and $2 \mu \mathrm{l}$ of primary PCR product as a template.

The uniplexed primary msp 1 final reaction conditions were same as in $m s p 2$. The three nested $m s p 1$ familyspecific amplification for Ro33-, K1- and MAD20families each contained $1 \mathrm{x}$ PCR buffer B, $2 \mathrm{mM} \mathrm{MgCl}_{2}$, $200 \mu \mathrm{M}$ of each dNTP, 0.4 units of Taq DNA polymerase, $25 \mu \mathrm{M}$ of each oligonucleotide primer and $2 \mu \mathrm{l}$ of primary $m s p 1$ amplification product as a template.

Thermo cycling was done using the MJ Thermal Controller PTC-100 ${ }^{\mathrm{TM}}$ (MJ Research Inc. Watertown, USA). Temperature cycling parameters were: initial denaturation at $95^{\circ} \mathrm{C}$ for 5 minutes followed by 30 cycles of denaturation at $94^{\circ} \mathrm{C}$ for 1 minute, annealing at $58^{\circ} \mathrm{C}$ for 2 minutes (for both uniplexed and duplexed primary PCR amplification) or $59^{\circ} \mathrm{C}$ for 2 minutes (for $m s p 1$ and glurp nested PCR) and extension at $72^{\circ} \mathrm{C}$ for 2 minutes followed by 10 minutes of final extension at $72^{\circ} \mathrm{C}$. Nested PCR for $m s p 2$ : initial denaturation was $94^{\circ} \mathrm{C}$ for 2 minutes followed by 30 cycles of denaturation at $94^{\circ} \mathrm{C}$ for 30 seconds, annealing at $50^{\circ} \mathrm{C}$ for 45 seconds and extension at $70^{\circ} \mathrm{C}$ for 2 minutes. The last extension cycle was prolonged for 10 minutes.

The primary and nested PCR amplification of the msp1 and glurp loci were carried out at IHI laboratory but all gel electrophoresis and digital image analysis were done at the Swiss TPH laboratory. For the $m s p 2$ locus, all primary amplification reactions were done at IHI laboratories whereas all nested PCR amplification and subsequent analysis were done in Basel and the conditions were as described elsewhere [19].

\section{Allele detection and estimation of molecular weight}

The nested glurp and $m s p 1$ PCR products were separated directly on $3 \%$ or $3.5 \%$ ethidium bromide-stained agarose gels, respectively, visualized under UV illumination and photographed. Gel photographs were visually examined to identify successfully amplified fragment. All glurp and mspl positive samples were separated again using $1.5 \%$ or $2 \%$ agarose gels, respectively, and directly analysed by a digitalized gel documentation system where band sizing and molecular weight were calculated by the AlphaEase ${ }^{\mathbb{R}} \mathrm{FC}$ software version 6.0.0 Alpha Innotech. The fragments were then grouped into "bins" differing by 25 bp for $m s p 1$ and 50 bp for glurp. All fragments falling within the limits of the bin were considered to belong to the same genotype. Bins were determined manually and allelic frequencies were calculated by Stata v 9.0 (Stata Corporation Inc, Texas USA and Stat View version 5.0.1).

The nested $m s p 2$ amplification products were separated in $1.5 \%$ agarose gel in order to identify positive samples, which were subsequently prepared for capillary electrophoresis analysis. Briefly, PCR products were diluted with distilled water to the ratios 1:10, 1:20 and $1: 30$ according to the intensity of the bands in the nested PCR product. This was followed by the addition 10 ul of a 1:40 fluorescent size standard ROX-500 (Applied Biosystems) consisting of 16 fragments ranging for $50-500 \mathrm{bp}$ in length, dried and shipped to the Medical Research Center Genomic Facility-London, England) for capillary electrophoresis. At the genomic facility, samples were analyzed by automated 3730xls DNA analyzer and a GeneMapper ${ }^{\circledR}$ software analyzer version 3.2. (Applied Biosystems LTD) was used to determine the size of PCR fragments, as previously described [19]. Analysis of $m s p 2$ raw data, allele calling according to manually selected $3 \mathrm{bp}$ bins and calculation of a cut off and allelic frequencies was performed by dedicated software designed at the Swiss TPH.

\section{Allelic richness and diversity and mean multiplicity of} infection (MOI)

As a measure for genetic diversity, the expected heterozygosity $\left(\mathrm{H}_{\mathrm{E}}\right)$ was calculated by use of the formula $\mathrm{H}_{\mathrm{E}}=[\mathrm{n} /(\mathrm{n}-1)]\left[\left(1-\sum \mathrm{P}_{\mathrm{i}}{ }^{2}\right)\right]$, where $\mathrm{n}=$ sample size, $\mathrm{Pi}=$ 
allele frequency. The mean multiplicity of infection (MOI) was calculated as the quotient of the total number of $P$. falciparum genotypes for each marker in a particular country and the number of positive PCR samples.

\section{Results}

\section{Study profile}

Out of 780 baseline (Day 0) DNA samples, 599 (76.8\%) were successfully amplified for $m s p 1,679$ (87\%) for msp2 and 575 (73.7\%) for glurp loci (Table 2).

\section{Mean multiplicity of infection (MOI)}

Table 3 shows mean MOIs calculated using data from each of the three marker genes for each of the five study countries. The mean MOI for $m s p 2$ loci was the highest (2.24), followed by that for msp1 (1.48) and then for glurp (1.4). The mean MOI values calculated for msp1 and glurp were similar but substantially different from those of $m s p 2$.

\section{Genetic diversity and allelic frequency}

The number of genotypes observed at each marker by country is shown in Table 4. Seventeen (17) different msp1 genotypes were observed, representing K1 (8 genotypes), MAD20 (8 genotypes) and RO33 (1 genotype) allelic families. The msp 1 fragment sizes ranged from 135 bp - 305 bp. Figure 1 shows the frequencies of msp1 genotypes by country. The majority of these genotypes occurred at a frequency below $10 \%$. However, four genotypes from the K1 allelic family (176 bp-201 bp, $201 \mathrm{bp}-2262 \mathrm{bp}, 226-251 \mathrm{bp}, 151-176 \mathrm{bp})$, two from the MAD20 family (176 bp-201 bp and $201 \mathrm{bp}-226 \mathrm{bp}$ ) occurred above $10 \%$.

The R033 family is considered to be monomorphic with a predicted amplified fragment size of $215 \mathrm{bp}$. However, the gel analysis software estimated the fragment amplified from most samples to be between 180 $\mathrm{bp}-210 \mathrm{bp}$. When a set of 6 samples representative of these two sizes were sequenced, they were all found to be 215 bp long.
The $m s p 2$ diversity and genotype frequencies for each of the countries are shown in Figure 2. A total of 116 different $m s p 2$ genotypes (size range from 205 to 518 bp) were recorded at the 5 study sites, of which, 83/116 (71.6\%) and 33/116 (28.4\%) belonged to the 3D7 and FC27 allelic families, respectively (Table 4). The frequencies of individual $m s p 2$ genotypes were low with $96.5 \%$ occurring at a frequency $\leq 5 \%$. Only four $(3.5 \%)$ genotypes, all belonging to the FC27 allelic family were found at frequencies at or above $5 \%$.

Fourteen (14) different glurp genotypes were detected and their diversity across the countries is shown in Figure 3. The allelic variants ranged between $650 \mathrm{bp}$ and $1,250 \mathrm{bp}$, and the majority (77.4\%) occurred at frequencies $<10 \%$. Two genotypes, bin sizes 907 bp-957 bp and $957 \mathrm{bp}-1,007 \mathrm{bp}$, had the highest frequencies, $20 \%$ in Uganda and 18\% in Malawi, respectively.

Expected heterozygosity $\left(\mathrm{H}_{\mathrm{E}}\right)$ was calculated in order to assess the discriminatory power of the three individual markers. $\mathrm{H}_{\mathrm{E}}$ estimates the fraction of all parasites that would be heterozygous for any of the three loci. Table 5 shows generally very high $\mathrm{H}_{\mathrm{E}}$ values across the five sites studies.

\section{Discussion}

Polymorphic regions from of the P. falciparum msp1, msp 2 and glurp loci have been selected as the recommended markers for parasite genotyping in anti-malarial drug trials and efficacy studies [23]. However, the parasites' genetic profile has not been systematically documented in many malaria endemic countries. A large number of archived P. falciparum positive pre-treatment infections were genotyped in order to compare the diversity and allelic frequencies for these three markers among five geographical areas with different transmission intensities across sub-Saharan Africa; namely Malawi, Tanzania, Uganda, Burkina Faso and São Tomé. These blood samples were collected in the region during two clinical trials (WHO/TDR and IHI/Swiss TPH) of artemisinin-based combination therapy that were conducted between 1996 and 2000. The findings from these

Table 2 DNA amplification success rate for each marker gene in baseline samples in 5 countries

\begin{tabular}{|c|c|c|c|c|c|c|c|c|}
\hline \multirow[t]{3}{*}{ Country } & \multirow[t]{3}{*}{ Study year } & \multirow[t]{3}{*}{$\mathbf{n}$} & \multicolumn{6}{|c|}{ PCR-positive samples } \\
\hline & & & \multicolumn{4}{|c|}{ msp1 (\%) } & \multirow[t]{2}{*}{ msp2 (\%) } & \multirow[t]{2}{*}{ glurp $(\%$} \\
\hline & & & K1 & MAD20 & R033 & Total & & \\
\hline $\mathrm{B}^{*}$. Faso & 2000 & 180 & 71 & 33 & 37 & $95(53)$ & $154(86)$ & $110(61)$ \\
\hline Malawi & 2000 & 180 & 100 & 53 & 68 & $146(81)$ & $145(81)$ & $135(75)$ \\
\hline São Tomé & 2000 & 180 & 100 & 65 & 65 & $171(95)$ & $164(91)$ & $145(81)$ \\
\hline Tanzania & 1996 & 60 & 48 & 30 & 10 & $50(83)$ & $56(93)$ & $45(75)$ \\
\hline Uganda & 2000 & 180 & 100 & 41 & 27 & $137(76)$ & $160(89)$ & $140(78)$ \\
\hline Total & & 780 & & & & 599(76.8) & 679 (87) & $575(73.7)$ \\
\hline
\end{tabular}

Note: ${ }^{*} \mathrm{~B}=$ Burkina, $\mathrm{S}=$ Study. 
Table 3 Mean Multiplicity of infection recorded using msp1, msp2 and glurp loci in the 5 study countries

\begin{tabular}{lllllll}
\hline & Malawi & B. Faso & Sao Tome & Tanzania & Uganda & Average \\
\hline msp1 & 1.03 & 1.40 & 1.30 & 2.50 & 1.18 & 1.48 \\
msp2 & 1.52 & 3.03 & 2.00 & 3.48 & 1.17 & 2.24 \\
glurp & 1.01 & 1.86 & 1.01 & 1.84 & 1.29 & 1.40 \\
\hline
\end{tabular}

Study participant were children 0 to 5 years old at all sites.

Note: $\mathrm{B}=$ Burkina.

studies have been published elsewhere [22,13]. The aim of this study was to determine whether the genetic diversity of the markers or their suitability for PCR-correction of drug efficacy trials in endemic countries, varied between countries. In addition, since the PCRcorrected treatment failures in both of the above trials were $>10 \%$, the study also aimed at validating these corrections by assessing the Day 0 genotypic profile as recommended [23].

Although Isocode stix were stored at room temperature over nine years, the majority (53 - 95\%) of the DNA samples could be amplified; with some unexplained variation unrelated to amplified fragment size across the geographical sites. This molecular genotyping study shows that on average the majority of the patients were infected with more than one parasite genotype on the day of admission. The mean MOI values were heterogenous across the different sites, being lowest in Uganda, and highest in Burkina-Faso and Tanzania. Mean MOI was highest for $m s p 2$ (2.24), followed by msp1 (1.48) and was lowest for glurp (1.40) and the allelic diversity followed a similar trend recording 116, 17 and 14 alleles, respectively. The allelic variants were spatially distributed across the five Sub-Saharan African countries. The differences in allele diversity between msp 2 on the one hand and msp 1 and glurp on the other are clearly attributable to the method used for DNA fragment sizing. Indeed capillary electrophoresis used for $m s p 2$ has a much higher power of resolution than gel elecrophoresis and digitalized fragment sizing used for msp1 and glurp [24]. However, since the genotyping methods used were the same for all geographic sites, it is possible to compare diversity of a given marker between countries. Tanzania recorded the highest genetic diversity, while Uganda recorded the lowest diversity in all the three markers.

In the WHO/TDR trials [15], the crude post day-14 parasitological recurrence rate for all treatment groups was $22 \%$ and was $89.7 \%$ for chloroquine alone and $13.6 \%$ for artemether-lumefantrine for the IHI-Swiss TPH trial [21]. According to the recently adopted recommendations for malaria genotyping [23], PCR-corrected failure rates exceeding $10 \%$ would require determining MOI and allelic frequencies in order to confirm the validity of the PCR-correction. Thus observation of high MOI, high allele diversity and low allele frequencies for all markers (especially msp 2 whose fragments were sized by the most accurate method) and study sites are strongly indicative of the high discriminatory power of this three-marker genotyping strategy. These findings further validate the PCR-adjusted outcomes recorded previously $[15,21]$.

High genetic diversity and low allelic frequencies have been reported previously from other sites that differ substantially in transmission intensity: Gabon [7], Uganda [10], Senegal [25], Burkina Faso [26] and Honduras [27]. Nonetheless, three allelic variants of msp1 (151-176 bp, 176-201 bp and 201-226 bp), four of $m s p 2$ (wos12, wos3, K1-long and FC27-455) and five of glurp (857-907 bp, 907-957 bp, 957-1007 bp, 10071057 and 1057-1107 were recorded at frequencies exceeding $5 \%$ in some of the study sites. Such frequently occurring genotypes might lead to misclassification of recurrent parasites in clinical trials and drug efficacy studies. However, from the current data even the most abundant genotype of glurp with a recorded allelic frequency of $20 \%$, the probability of acquiring a new independent infection of the same genotype by chance equals the square of the allelic frequency. This probability of $4 \%$ for a reinfection with the same genotype is not very high for a single genotyping marker; it would be even much lower when all three markers were genotyped.

Table 4 msp1, msp2 and glurp alleles observed in the 5 study countries

\begin{tabular}{lcccccccc}
\hline Country & \multicolumn{7}{c}{ Number of genotypes per allelic-family/locus } \\
\hline & K1 (\%) & Mad20 (\%) & RO33 (\%) & Total & 3D7 (\%) & FC27 (\%) & Total & glurp \\
\hline B**. Faso & $8(53.3)$ & $6(40)$ & $1(6.7)$ & 15 & $71(73.2)$ & $26(26.8)$ & 97 \\
São Tomé & $8(50)$ & $7(43.7)$ & $1(6.3)$ & 16 & $60(80)$ & $15(20)$ & 75 & 14 \\
Malawi & $7(53.8)$ & $5(38.5)$ & $1(7.7)$ & 13 & $53(76.8)$ & $16(23.2)$ & 69 & 13 \\
Uganda & $5(50)$ & $4(40)$ & $1(10)$ & 10 & $38(77.6)$ & $11(22.4)$ & 49 & 10 \\
Tanzania & $8(50)$ & $7(43.8)$ & $1(6.20)$ & 16 & $57(78.1)$ & $16(21.9)$ & 73 & 13 \\
\hline Total* & 8 & 8 & 1 & $\mathbf{1 7}$ & 83 & 33 & $\mathbf{1 1 6}$ & $\mathbf{1 4}$ \\
\hline
\end{tabular}

Note: ${ }^{*}$ Number of observed allele not sum of alleles, ${ }^{* *} B=$ Burkina. 


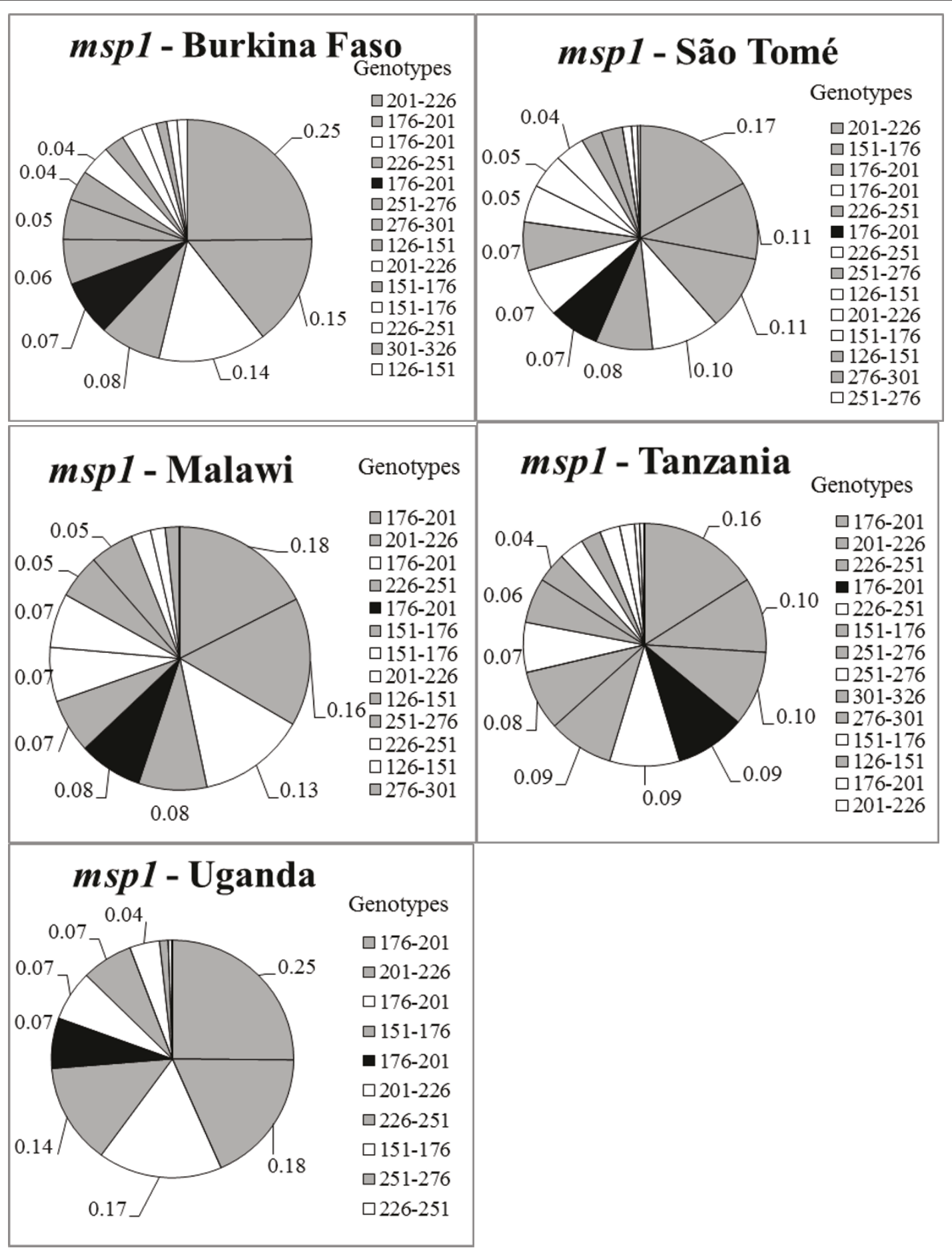

Figure $1 \mathrm{msp} 1$ allelic frequency (\%) by country. Most of the genotypes occurred below 10\%. Four K1-family genotypes and two MAD20family genotypes occurred above $10 \%$. Note; only genotypes with frequency $\geq 4 \%(0.04)$ are labeled.

It should be noted that the blood samples analysed were collected more than nine years ago, hence the genetic profiles described might no longer accurately represent the current situation. Nonetheless, this study provides important genetic background data in these areas. The strength of the present study hinges on four major factors: (i) the large number of baseline samples that, (ii) were collected from five countries with different levels of transmission intensity, (iii) use of automated DNA sizing methods to remove investigator bias and 


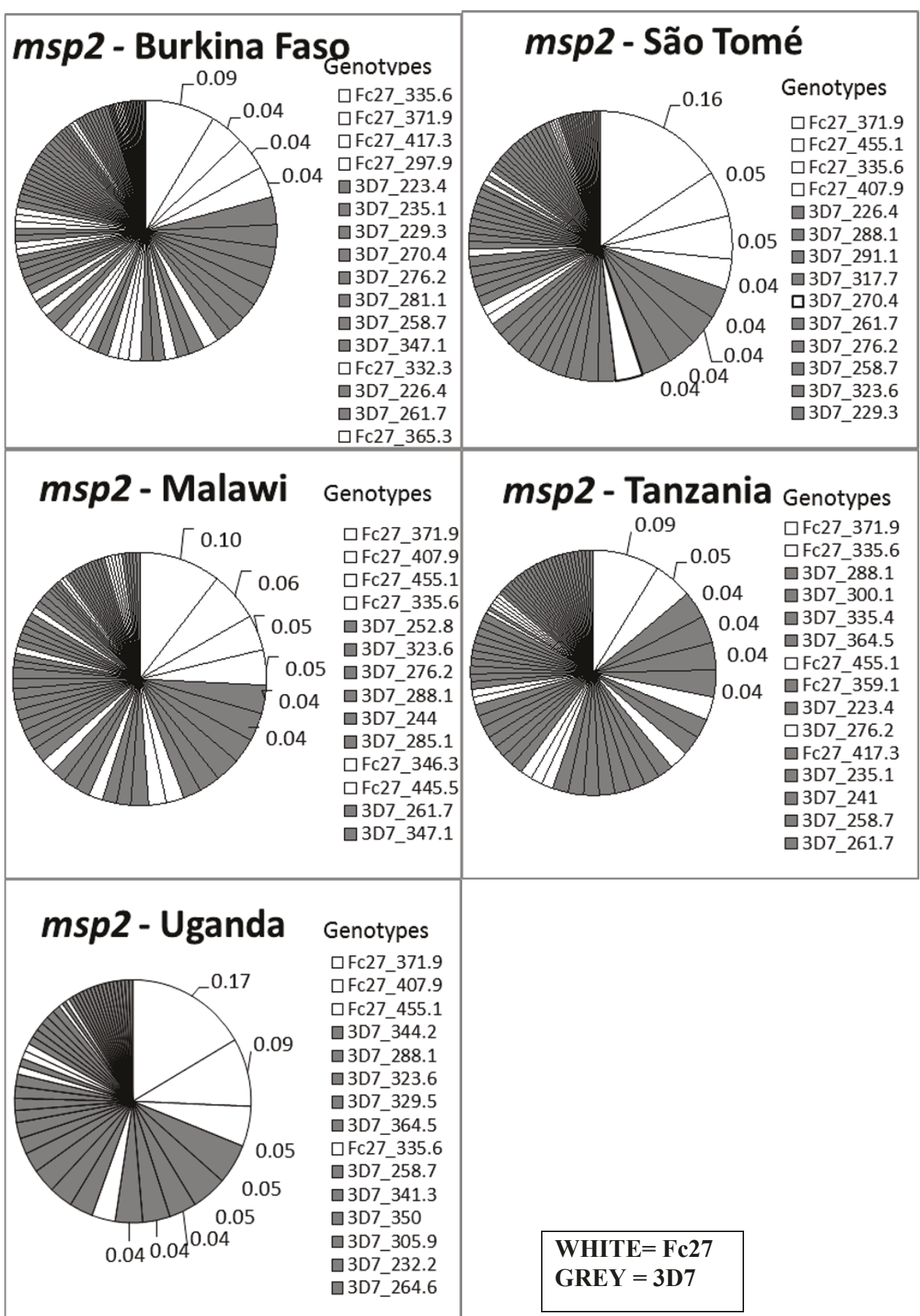

Figure 2 msp2 allelic diversity and frequency (\%) by country. Only three genotypes, all from FC27 family occurred at of above 5\%. Note; only genotypes with frequency $\geq 4 \%(0.04)$ are labeled.

error in assigning molecular weights of the PCR products, and (iv) comparative analyses across countries could be conducted, because the data was obtained using the same amplification protocol.

One of the factors that directly impinge on the utility of genotyping protocols in drug efficacy studies is that of bin size selection. For this study, as for all previous studies, the bin sizes for msp1 or glurp fragments have been set rather arbitrarily. The lengths of the repeat units, whose number varies between the different allelic variants, was taken into account to set bin width of $25 \mathrm{bp}$ and $50 \mathrm{bp}$ for $m s p 1$ and glurp, respectively. In contrast, $m s p 2$ fragments 


\section{glurp-Burkina Faso}
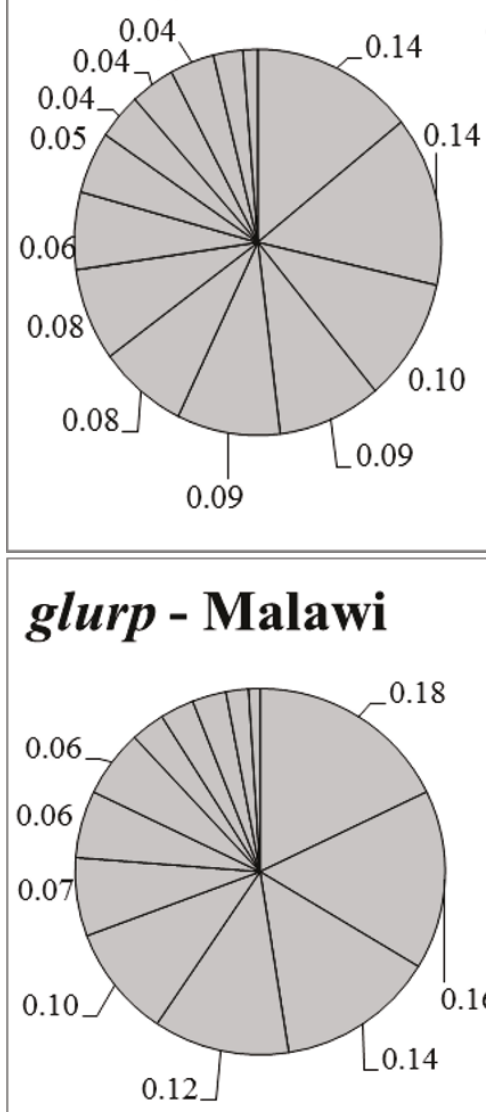

\section{glurp - Uganda}

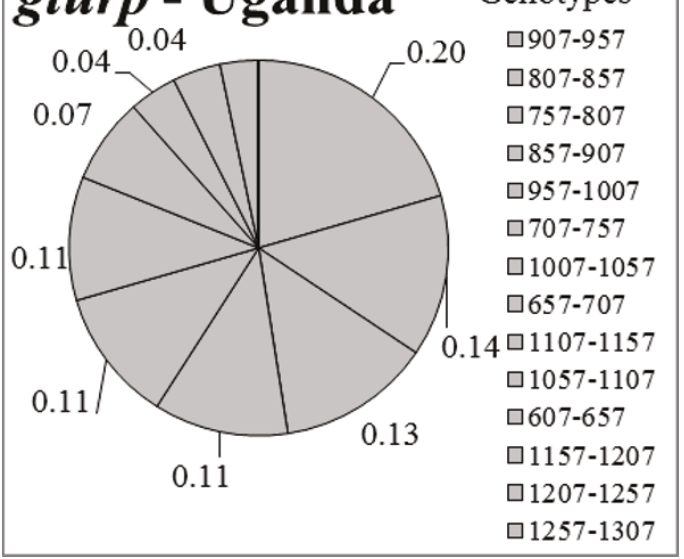

$\square 857-907$
Genotypes

$\square$ 1007-1057

$\square 807-857$

$\square 757-807$

$\square 957-1007$

口907-957

$\square$ 1057-1107

$\square 707-757$

$\square 1207-1257$

$\square 657-707$

1107-1157

ㅁ1157-1207

$\square 607-657$

$\square$ 1257-1307

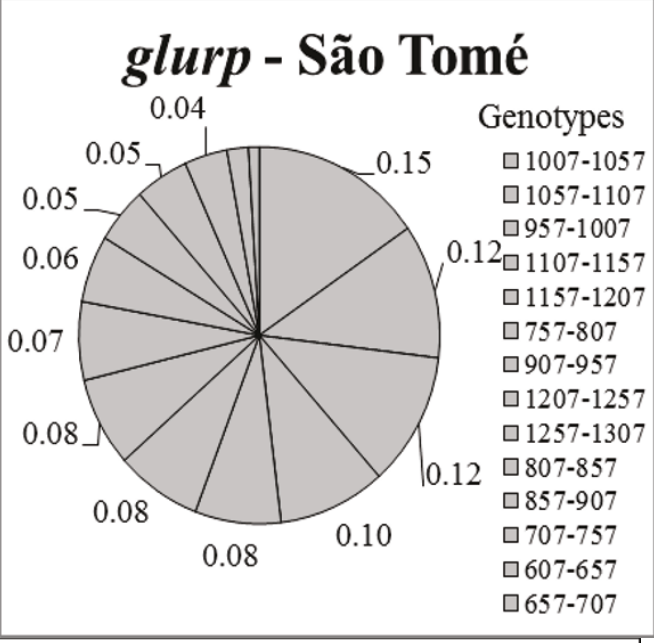

glurp -Tanzania

$\square$ 957-1007

$\square$ 1007-1057

$\square 907-957$

$\square$ 1057-1107

$\square 757-807$

$\square 707-757$

$\square 1107-1157$

$\square 657-707$

$0.16 \square 807-857$

$\square$ 1157-1207

$\square 1207-1257$

$\square 607-657$

$\square$ 1257-1307

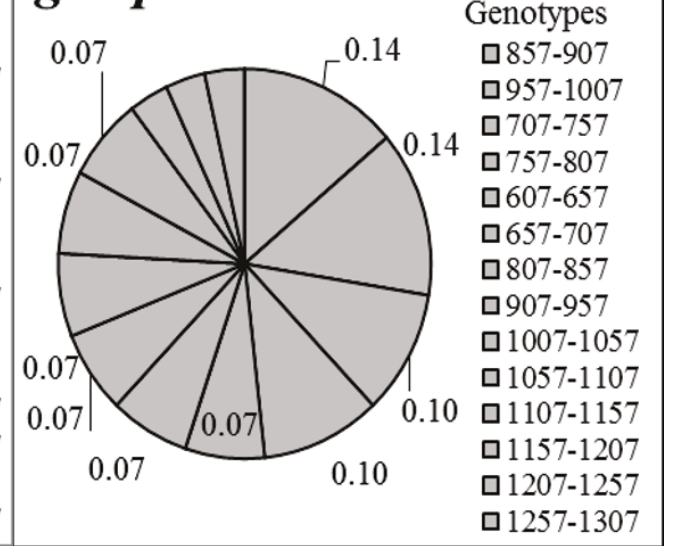

Figure 3 glurp allelic diversity and frequency (\%) by country. Only genotypes with frequency $\geq 4 \%(0.04)$ are labeled.

were sized by capillary electrophoresis with 3 bp bin width that quite obviously represents the smallest size difference possible in a coding region. In previous studies, different bin widths were used, for example, [28] used a conservative bin width of $40 \mathrm{bp}$ for $m s p 1$ and $m s p 2$, whilst [10] used bin sizes of $10 \mathrm{bp}$ for $m s p 1$ and $m s p 2$ and $20 \mathrm{bp}$ for glurp. In addition to these variations in bin width, the different fragment sizing methods employed by different researchers make it difficult to compare data for a particular marker between studies. 
Table 5 Genetic diversity of $m s p 1$, msp2 and glurp measured as expected heterozygosity $\left(\mathrm{H}_{\mathrm{E}}\right)$

\begin{tabular}{llllll}
\hline \multicolumn{5}{c}{$\mathrm{H}_{\mathrm{E}}=[\mathbf{n} /(\mathbf{n}-\mathbf{1})]\left[\left(1-\Sigma \mathbf{P}_{\mathbf{i}}{ }^{2}\right)\right]$} \\
& Malawi & Burkina Faso & São Tomé & Tanzania & Uganda \\
\hline msp2 & 0.97 & 0.98 & 0.96 & 0.99 & 0.95 \\
msp1 & 0.79 & 0.78 & 0.83 & 0.84 & 0.68 \\
glurp & 0.89 & 0.91 & 0.91 & 0.92 & 0.88 \\
\hline
\end{tabular}

Another drawback of this genotyping protocol is the variability in the electrophoretic migration of a given DNA fragment between gels, and indeed between different regions of the same gel. This is clearly illustrated in our observations of variable RO33 fragment sizes by digital gel documentation, despite the fact that these fragments were confirmed to be of the same size (215 bp) by sequencing. It is most likely that such spurious variability will also occur for variants of the other $m s p 1$ allelic families, and maybe even to a greater extent in the much larger glurp fragments. Thus, the quality and value of the data obtained from binning of alleles across different gels depends largely on gel quality (e.g. "smiling effect" or unequal loading of gel slot) and on the accuracy of image digitization. Electrophoretic variability is a potential problem when one wishes to establish the frequencies of each allelic variant. When sizing is done across different gels, binning through an image analyzer might be more prone to error than doing it by eye, especially when samples are run side by side. This error observed when comparing fragments from different gels, is highly unlikely to affect the validity of the genotype pattern comparisons of baseline and recurrent infections, on which PCR correction is based, because the amplified products from these samples are usually migrated in the gels side-by-side and, therefore, with a much reduced chances of variability. For such comparisons, detection of size differences can either be done visually or through an image analysis programme. Admittedly, visual analysis also poses some degree of subjectivity: at what level of difference in migration does one say that two bands are different, especially when they differ in quantity and consequently intensity and thickness in the gel.

Ultimately, the ability to distinguish between two allelic variants depends directly on the resolution of the method used to analyse the amplified fragments. At present capillary electrophoresis offers the best solution, because it provides accurate and reproducible estimates of DNA fragment lengths with a resolution power down to a few base pairs difference. It is highly likely that the $m s p 1$ and glurp fragments amplified in the course of this study in fact encompassed a larger repertoire of distinct allelic variants than those resolved by simple agarose gel electrophoresis. By the time this study was done, only $m s p 2$ capillary electrophoresis protocol was described [19]. Now protocols for msp1 [29,30] and glurp [24] have been developed and all three markers can be analyzed and allele diversity/frequency compared more accurately.

\section{Conclusion}

The $P$. falciparum msp1, msp 2 and glurp markers used for PCR-correction of treatment outcomes in the context of drug clinical trials or efficacy studies in endemic areas appear to be highly polymorphic and to have low allelic frequencies across sub-Saharan countries with varying transmission intensities. These observations reinforce the value of these genotyping markers in classifying recurrent post-treatment $P$. falciparum episodes as recrudescence or new-infections. However, these findings strongly suggest that standardized protocols should include optimal methods for fragment size estimation and bin width determinations. This will further enable and simplify data comparison between sites/studies. With the expanding access to ACT and current changes in malaria epidemiology, the $P$. falciparum $m s p 1, m s p 2$ and glurp allele frequency/genetic diversity should be monitored regularly to ensure the reliability of the PCR-adjusted treatment outcome.

\section{Acknowledgements}

These studies were funded by UNICEF/UNDP/World Bank/WHO Special program for Research and Training in Tropical Diseases (TDR) and Novartis pharmaceutical company. $1 \mathrm{H} \|$ receives core financial support from the Swiss Agency for Development and Co-operation (SDC) and Department for International Development (DFID). Felista Mwingira's MSc project was jointly sponsored by TDR, IHI and Dar Es Salaam University College of Education (DUCE), a constituent college of the University of Dar es salaam - Tanzania. We would like to extend our thanks to the field teams in the African countries. We are also grateful to the $\mathrm{IH}$ laboratory technicians and attendants who were involved in one way or another in genotyping. Last but not least, we are thankful to parents/guardians of all children who volunteered and consented to participate in these trials.

Disclaimer

The views expressed in this article are those of the authors and not of their institutions. P. Olliaro is a staff member of the WHO; the authors alone are responsible for the views expressed in this publication and they do not necessarily represent the decisions, policy or views of the WHO.

\section{Author details}

'Dares Salaam University College of Education P.O.BOX 2329, Dar es Salaam, Tanzania. ${ }^{2}$ Department of Zoology and Wild life Conservation, University of Dar es Salaam, PO Box 35064, Dar es Salaam, Tanzania. ${ }^{3}$ Swiss Tropical and Public Health Institute, Socinstrasse 57, CH 4002, Basel, Switzerland. ${ }^{4}$ University of Basel, Petersplatz 1, 4003 Basel, Switzerland. ${ }^{5}$ ffakara Health Institute, P. O. Box 74 Bagamoyo, Tanzania. ${ }^{6}$ UPMC/INSERM UMR S 945, Faculté de Médecine Pitié-Salpêtrière Université Pierre \& Marie Curie, 91 Boulevard de l'Hôpital, 75013 Paris, France. ${ }^{7}$ UNICEF/UNDP/World Bank/WHO Special Programme for Research and Training in Tropical Diseases, World Health Organization, Room CA 1118, Centre Casai, 51-53 Avenue Louis Casai1216 Cointrin, Geneva, Switzerland. ${ }^{8}$ Centre for Tropical Medicine and Vaccinology, Nuffield Department of Medicine, University of Oxford, Churchill Hospital, Oxford OX37LJ, UK

\section{Authors' contributions}

Study conceptualization and design was done by KM, HPB and PO. KM and IF did study monitoring and supervision in Tanzania and Switzerland, 
respectively. IF and GS provided technical advisory support in genotyping and data interpretation. FM, DS, SS and KM carried out the laboratory work. FM and GN performed statistical analysis. FM, GN, and KM composed the primary version of the manuscript and all other authors contributed modifications. All authors read and approved the final manuscript.

\section{Statement of conflict of interest}

The authors do not have any commercial or other association that may pose conflicts of interest concerning the work reported in this paper.

Received: 22 October 2010 Accepted: 6 April 2011

Published: 6 April 2011

\section{References}

1. Stepniewska K, Taylor WR, Mayxay M, Price R, Smithuis F, Guthmann JP, Barnes K, Myint HY, Adjuik M, Olliaro P, Pukrittayakamee S, Looareesuwan S, Hien TT, Farrar J, Nosten F, Day NP, White NJ: In vivo assessment of drug efficacy against Plasmodium falciparum malaria: duration of follow up. Antimicrob Agents Chemother 2004, 48:4271-4280.

2. Felger I, Smith T, Edoh D, Kitua A, Alonso P, Tanner M, Beck HP: Multiple Plasmodium falciparum infection in Tanzanian infants. Trans $R$ Soc Trop Med Hyg 1999, 93:29-34.

3. Babicker HA, Lines J, Hill WG, Walliker D: Population structure of Plasmodium falciparum in villages with different malaria endemicity in East Africa. Am J Trop Med Hyg 1997, 56:141-147.

4. Snounou G, Zhu X, Siripoon N, Jarra W, Thaithong S, Brown KN, Viriyakosol S: Biased distribution of MSP-1 and MSP-2 allelic variants in Plasmodium falciparum populations in Thailand. Trans $R$ Soc Trop Med Hyg 1999, 93:369-374.

5. Peyerl-Hoffmann G, Jelinek T, Kilian A, Kabagambe G, Metzger WG, von Sonnenburg F: Genetic diversity of Plasmodium falciparum and its relationship to parasite density in an area with different malaria endemicity in West Uganda. Trop Med Int Health 2001, 6:607-613.

6. Magesa SM, Mdira KY, Farnert A, Simionsen PE, Bygbjerg IC, Jakobsen PH: Distinguishing Plasmodium falciparum treatment failures from reinfections by using PCR genotyping in a holoendemic area in Northeastern Tanzania. Am J Trop Med Hyg 2001, 65:477-483.

7. Auboy A, Migot-Nabias F, Deloron P: Polymorphism in two merozoite surface protein of Plasmodium falciparum isolates from Gabon. Malar $\mathrm{J}$ 2003, 2:12.

8. Snounou G, Beck HP: The use of PCR genotyping in the assessment of recrudescence or re-infection after antimalarial drug treatment. Parasitol Today 1998, 14:462-467.

9. Beck HP: How does molecular epidemiology help to understand malaria? Trop Med Int Health 1999, 4:1-3.

10. Cattamanchi A, Kyabayinze D, Hubbard A, Rosenthal PJ, Dorsey G: Distinguishing recrudescence from re-infection in a longitudinal antimalaria drug efficacy study: comparison of results based on genotyping of msp 1, msp 2 and glurp. Am J Trop Med Hyg 2003, 68:133-139.

11. Happi CT, Gbotosho GO, Sowunmi A, Falade CO, Akinboye DO, Gerena L, Kyle DE, Milhous W, Wirth DF, Oduola AMJ: Molecular analysis of plasmodium falciparum recrudescent malaria infections in children treated with chloroquine in Nigeria. Am J Trop Med Hyg 2004, 70:20-26.

12. Adjuik M, Agnamey P, Babiker A, Borrmann S, Brasseur P, Cisse M, Cobelens F, Diallo S, Faucher JF, Garner P, Gikunda S, Kremsner PG, Krishna S, Lell B, Loolpapit M, Matsiegui PB, Missinou MA, Mwanza J, Ntoumi F, Olliaro P, Osimbo P, Rezbach P, Some E, Taylor WR: Amodiaquine-artesunate versus amodiaquine for uncomplicated Plasmodium falciparum malaria in African children: a randomized multicentre trial. Lancet 2002, 359:1365-1372.

13. Adjuik M, Babiker A, Garner P, Olliaro P, Taylor W, White N, International Artemisinin Study Group: Artesunate combinations for treatment of malaria: meta-analysis. Lancet 2004, 363:9-17.

14. Mutabingwa TK, Anthony D, Heller A, Hallett R, Ahmed J, Drakeley C, Greenwood BM, Whitty CJ: Amodiaquine alone, amodiaquine +sulfadoxine-pyrimethamine, amodiaquine+artesunate, and artemetherlumefantrine for outpatient treatment of malaria in Tanzanian children: a four-arm randomised effectiveness trial. Lancet 2005, 365:1474-1480.

15. Mugittu K, Adjuik M, Snounou G, Ntoumi F, Taylor W, Mshinda H, Olliaro P, Beck HP: Molecular genotyping to distinguish between recrudescents and new infections intreatment trials of Plasmodium falciparum malaria conducted in Sub-Saharan Africa: adjustment of parasitological outcomes and assessment of genotyping effectiveness. Trop Med Int Health 2006, 9:1350-1359.

16. Mugittu K, Priotto G, Guthmann JP, Kiguli J, Adjuik M, Snounou G, Beck HP, Mshinda H, Olliaro PL, Taylor WRJ: Molecular genotyping in a malaria treatment trial in Uganda- unexpected high rate of new infections within two weeks after treatment. Trop Med Int Health 2007, 12:219-223.

17. Sirima SB, Tiono AB, Gansane A, Diarra A, Ouedraogo A, Konate AT, Kiechel JR, Morgan CC, Olliaro PL, Taylor WRJ: African children with acute uncomplicated Plasmodium falciparum. Malar J 2009, 8:48.

18. Flueck C, Frank G, Smith T, Jafarshad A, Nebie I, Sirima SB, Olugbile $S$, Alonso P, Tanner M, Druilhe P, Felger I, Corradin G: The risk of malarial infections and disease in Papua New Guinean children. Am J Trop Med Hyg 2007, 76:997-1008

19. Falk N, Marie N, Sama W, Owusu-Agyei S, Smith T, Beck HP, Felger I: Comparison of PCR and gene scan-based genotyping for analyzing infection dynamics of Plasmodium falciparum. Am J Trop Med Hyg 2006, 74:944-950.

20. Silue KD, Felger I, Utzinger J, Beck HP, Smith TA, Tanner M, N'goran EK: Prevalence, genetic diversity and multiplicity of Plasmodium falciparum infections in school children in central cote d'Ivore. Med Trop 2006, 66:149-156.

21. Hatz C, Abdulla S, Mull R, Schellenberg D, Gathmann I, Kibatala P, Beck HP Tanner M, Royce C: Efficacy and safety of CGP 56697 (artemether and benflumetol) compared with chloroquine to treat acute falciparum malaria in Tanzanian children aged 1-5 years. Trop Med Int Health 1998, 3:498-504

22. Irion A, Felger I, Abdulla S, Smith T, Mull R, Tanner M, Hatz C, Beck HP: Distinction of recrudescence from new infections by PCR-RFLP analysis in a comparative trial of cgp 56697 and chloroquine in Tanzanian children. Trop Med Int Health 1998, 3:490-497, Trop Med Int Health. 19983 490-494.

23. Beck HP, Felger I: Methods and techniques for clinical trials on antimalarial drug efficacy: genotyping to identify parasite population Amsterdam, The Netherlands, Geneva: Medicines for Malaria Venture; 2007, 45, Informal consultation organized by the Medicines for Malaria Venture and cosponsored by the World Health Organization.

24. Gupta V, Dorsey G, Hubbard AE, Rosenthal PJ, Greenhouse B: Gel versus capillary electrophoresis genotyping for categorizing treatment outcomes in two anti-malarial trials in Uganda. Malar J 2010, 9:19.

25. Henry M, Diallo I, Bordesj, Ka S, Pradines B, Diatta B, M'baye PS, Sane M, Thiam M, Gueye Mp, Wade B, Touze Je, Debonne Jm, Rogier C, Fusai T: Urban malaria in Dakar, Senegal: chemosusceptibility and genetic diversity of Plasmodium Falciparum isolates. Am J Trop Med Hyg 2006, 75:146-151.

26. Soulama I, Nébié I, Ouédraogo A, Gansane A, Diarra A, Tiono AB, Bougouma EC, Konaté AT, Kabré GB, Taylor WRJ, Sirima S: Plasmodium falciparum genotypes diversity in symptomatic malaria of children living in an urban and a rural setting in Burkina Faso. Malar J 2009, 8:135.

27. Haddad D, Snounou G, Mattei D, Enamorad Gl, Figueroa J, Stahl S, Berzins K: Limited genetic diversity of Plasmodium falciparum in field isolates from Honduras. Am J Trop Med Hyg 1999, 60:30-34.

28. Zhong D, Afrane Y, Githeko A, Yang Z, Cui L, Menge DM, Temu EA, Yan G: Plasmodium falciparum genetic diversity in Western Kenya Highlands. Am J Trop Med Hyg 2007, 77:1043-1050.

29. Schoepflin S, Valsangiacomo F, Lin E, Kiniboro B, Mueller I, Felger I: Comparison of Plasmodium falciparum allelic frequency distribution in different endemic settings by high-resolution genotyping. Malar J 2009, 8:250.

30. Liljander A, Wiklund L, Falk N, Kweku M, Mårtensson A, Felger I, Färnert A: Optimization and validation of multi-coloured capillary electrophoresis for genotyping of Plasmodium falciparum merozoite surface proteins (msp1 and 2). Malar J 2009, 8:78.

doi:10.1186/1475-2875-10-79

Cite this article as: Mwingira et al:: Plasmodium falciparum msp1, msp2 and glurp allele frequency and diversity in sub-Saharan Africa. Malaria Journal 2011 10:79. 